\title{
Optical filter based on two coupled PhC GaAs-membranes
}

\author{
Tiziana Stomeo, ${ }^{1, *}$ Marco Grande, ${ }^{2}$ Gabriele Rainò, ${ }^{1,5}$ Adriana Passaseo, ${ }^{1}$ Antonella D'Orazio, ${ }^{2}$ \\ Roberto Cingolani, ${ }^{3}$ Andrea Locatelli, ${ }^{4}$ Daniele Modotto, ${ }^{4}$ \\ Costantino De Angelis, ${ }^{4}$ and Massimo De Vittorio ${ }^{1,3}$ \\ ${ }^{1}$ National Nanotechnology Laboratory of CNR/INFM, Distretto Tecnologico-ISUFI, Università del Salento, \\ Via Arnesano, 73100 Lecce, Italy \\ ${ }^{2}$ Dipartimento di Elettronica ed Elettrotecnica, Politecnico di Bari, Via Re David 200, 70125 Bari, Italy \\ ${ }^{3}$ Italian Institute of Technology (IIT), Center for Bio-Molecular Nanotechnology, Via Barsanti 1, \\ Arnesano, 73010 Lecce, Italy \\ ${ }^{4}$ Dipartimento di Elettronica per l'Automazione, Università degli Studi di Brescia, Via Branze 38, 25123 Brescia, Italy \\ ${ }^{5}$ Present address: IBM Research GmbH, Saümerstrasse 4, 8803 Rüschlikon, Switzerland \\ *Corresponding author: tiziana.stomeo@unisalento.it
}

Received October 6, 2009; revised December 16, 2009; accepted December 17, 2009; posted January 5, 2010 (Doc. ID 118244); published January 29, 2010

We demonstrate an ultracompact optical filter based on two coupled high-index contrast GaAs photonic crystal $(\mathrm{PhC})$ membranes. The PhC membranes consist of a square lattice of air holes and behave as a FabryPerot cavity whose reflectivity and transmissivity depend on the air gap between the two membranes. The normal-incidence reflectance measurements and the numerical simulation of reflection spectra show a high sensitivity to the geometrical parameters, such as the distance between the slabs, whose control would make the device suitable for a new class of tunable optical filters. (C) 2010 Optical Society of America

OCIS codes: $130.3120,220.3740,220.4241,230.5298,230.7400$.

The capability of photonic crystal $(\mathrm{PhC})$ structures to control the flow of light makes them very attractive for several applications in the field of integrated optics and telecommunications [1-3]. In recent years a large number of investigations showed the performance enhancement induced by $\mathrm{PhC}$ optical sensors $[4,5]$. The optical properties of such periodic media are determined by the interference of multiple diffracted waves and are very sensitive to the geometrical parameters and to the refractive index of the component media. Such a sensitivity can be exploited for the purposes of optical sensing in a number of different ways [6,7]. In many applications, the sensitive devices are fabricated on a single $\mathrm{PhC}$ slab [8] that has been demonstrated to be the ideal substrate for the large-scale integration of optical circuits and devices. Suh et al. [9] described an alternative approach based on two parallel $\mathrm{PhC}$ slabs and theoretically demonstrated that a strong variation in the transmissivity of light through such structures can be accomplished with only a nanoscale variation in the spacing between the slabs. This approach relies on the existence of guided resonances in the slabs: a guided resonance, as a guided mode, is characterized by a strong confinement of the optical energy inside the slab but, differently from the guided mode, the guided resonance allows a very efficient coupling with the external incoming light beam $[10,11]$. In theory, in order to switch the transmission coefficient of the double-layer structure from 1 to 0.01 , the variation in the spacing between the slabs is just a few percent of the operating wavelength [9]. Doublemembrane $\mathrm{PhC}$ cavities have also been investigated theoretically, leading to a novel approach for the optomechanical control of light and matter [12] and applications in the fluorescence enhancement and multi-analyte detection [13], but the proposed struc- tures have not yet been fabricated. In this Letter we report, for the first time to our knowledge, on the fabrication and the optical characterization of a GaAs coupled-membrane device that operates in the 1200$1300 \mathrm{~nm}$ wavelength range.

The layout of the fabricated device is shown in Fig. 1. The structure is composed of two PhC GaAsmembranes surrounded by air. Each membrane consists of a periodic array of air holes into the highindex guiding layer. A square lattice configuration is adopted for the two-dimensional (2D) PhC. Experimentally, we used a heterostructure with multiple etch-stop layers consisting (from top of the wafer to bottom) of a $376 \mathrm{~nm}$ GaAs-membrane layer, a $520 \mathrm{~nm}$ $\mathrm{Al}_{0.7} \mathrm{Ga}_{0.3} \mathrm{As}$ etch-stop layer, a $376 \mathrm{~nm}$ GaAsmembrane layer, and a $2000 \mathrm{~nm} \mathrm{Al}_{0.7} \mathrm{Ga}_{0.3} \mathrm{As}$ etchstop layer on a GaAs substrate. The heterostructure was grown by molecular beam epitaxy. Both the etchstop layers are sacrificial and they are removed to realize the two coupled membranes. A $400 \mathrm{~nm}$ hydrogen silsesquioxane resist layer was deposited on the sample as a hard mask for the high-aspect-ratio etching of $\mathrm{PhC}$ patterns [14]. The $2 \mathrm{D} \mathrm{PhC}$ pattern was written using a Raith 150 electron-beam (e-beam) lithography system operating at $30 \mathrm{kV}$. A thickness of $400 \mathrm{~nm}$ of the ZEP520-A resist was chosen to ensure

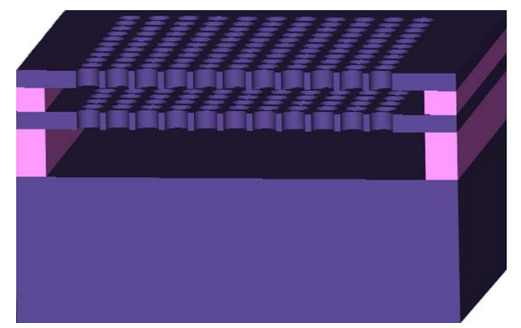

$A l_{0.7} G a_{0.3} A s$

Fig. 1. (Color online) Layout of the two coupled $\mathrm{PhC}$ GaAs-membranes. 
sufficient durability as a mask for the transfer of the pattern into the underlying spin-on glass hard mask and, at the same time, to ensure a good resolution of the e-beam writing. The key issue in the fabrication process is to achieve $\mathrm{PhC}$ devices with well-controlled geometrical feature sizes. A proximity error correction was applied to accomplish this target. Moreover, in order to achieve smooth circular holes with fast exposures, our e-beam lithography system was used in a "circular mode": in this way, every circular hole is exposed by the deflection of the beam along concentric circles. Different high-quality $\mathrm{PhC}$ patterns with well-controlled sizes were achieved after the resist development. The patterns defined in the ZEP resist layer were then transferred into the spin-on glass hard mask using an inductive coupled plasma (ICP) etching (AOE-STS) in fluorine chemistry until the GaAs surface was reached. In a following etching step, the holes-defining the square lattice configuration-were deep etched (more than 1300 $\mathrm{nm}$ ) through the two GaAs waveguide layers and into the sacrificial layer by $\mathrm{SiCl}_{4}$ ICP etching. The final step was the complete removal of the underlying sacrificial $\mathrm{Al}_{0.7} \mathrm{Ga}_{0.3} \mathrm{As}$ layers to realize the two $\mathrm{PhC}$ membranes. This target was achieved by using a $\mathrm{HF}: \mathrm{H}_{2} \mathrm{O}(1: 10)$ wet etching at room temperature [15] through the $\mathrm{PhC}$ holes and the trenches around the periodic pattern. Scanning electron microscope (SEM) pictures of the fabricated double-layer $\mathrm{PhC}$ devices are shown in Fig. 2. The regular square pattern of the $\mathrm{PhC}$ slab can be recognized in the middle of Fig. 2(a); the rectangular areas on the left and the right sides are the large trenches needed to remove the sacrificial layers by using wet etching. Highquality $\mathrm{PhC}$ patterns (464 $\mathrm{nm}$ hole diameter and 627 $\mathrm{nm}$ period) with well-controlled sizes were achieved after the pattern was transferred into the GaAs layers [Fig. 2(b)]. At the same time deep-etched holes and vertical sidewalls were also obtained [Fig. 2(c)]. Figure 2(d) shows the cross section of the final device,
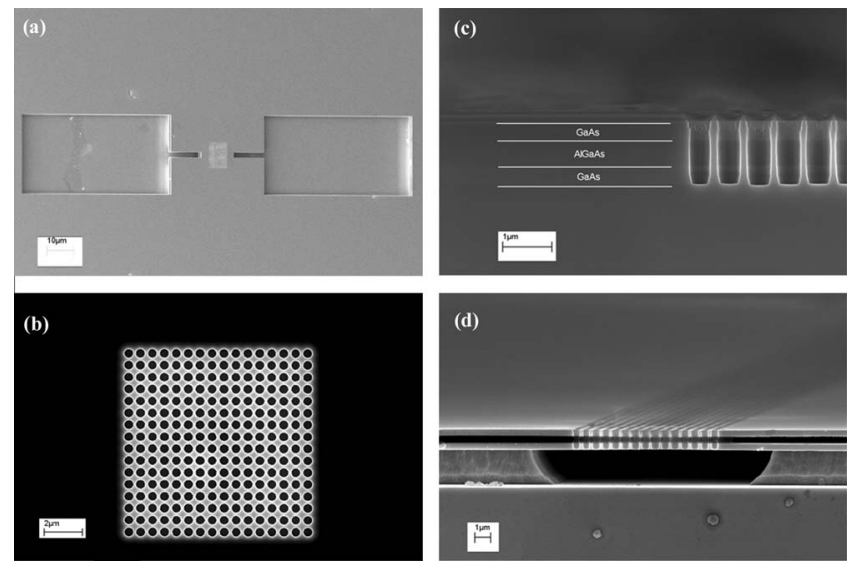

Fig. 2. SEM pictures of the fabricated double-layer $\mathrm{PhC}$ membranes: (a) bird's eye view (angle of $\sim 45^{\circ}$ ) of the complete device layout; (b) details of the $2 \mathrm{D}$ PhC pattern (464 $\mathrm{nm}$ hole diameter and $627 \mathrm{~nm}$ period); (c) cross section of the 2D PhC pattern after ICP process (hole depth is more than $1300 \mathrm{~nm}$ ); (d) coupled PhC GaAs-membranes surrounded by air after removal of the sacrificial layers. where the dark regions correspond to the empty regions, confirming the membrane release.

To optically test the double-membrane structure, reflectance measurements have been performed by using a $\mu$-reflectance setup. A light probe beam (from a tungsten broad band lamp) was focused onto the sample by a high-NA microscope objective. The reflected beam was then collected by the same microscope objective and measured by a monochromator coupled to a N-cooled InGaAs optical multichannel analyzer. The measured reflection spectra for two different hole diameters are reported in Fig. 3(a). The minimum reflectivity (i.e., the peak of transmissivity) is reached at 1200 and $1185 \mathrm{~nm}$ for the 459 and 464 $\mathrm{nm}$ hole diameter devices, respectively.

The reflectivity of the double-layer devices has been thoroughly simulated by means of a threedimensional (3D) finite-difference time-domain (FDTD) code. For the sake of comparison with the experimental results, we show in Fig. 3(b) the corresponding calculated reflectivity spectra. The numerical analysis confirms that the position of the resonance peak shifts toward longer wavelengths when the hole diameter is decreased. The calculated resonance wavelengths are roughly $40 \mathrm{~nm}$ greater than the measured ones; this difference can be ascribed to several factors, but we believe that some of them have a higher influence. In particular, a careful
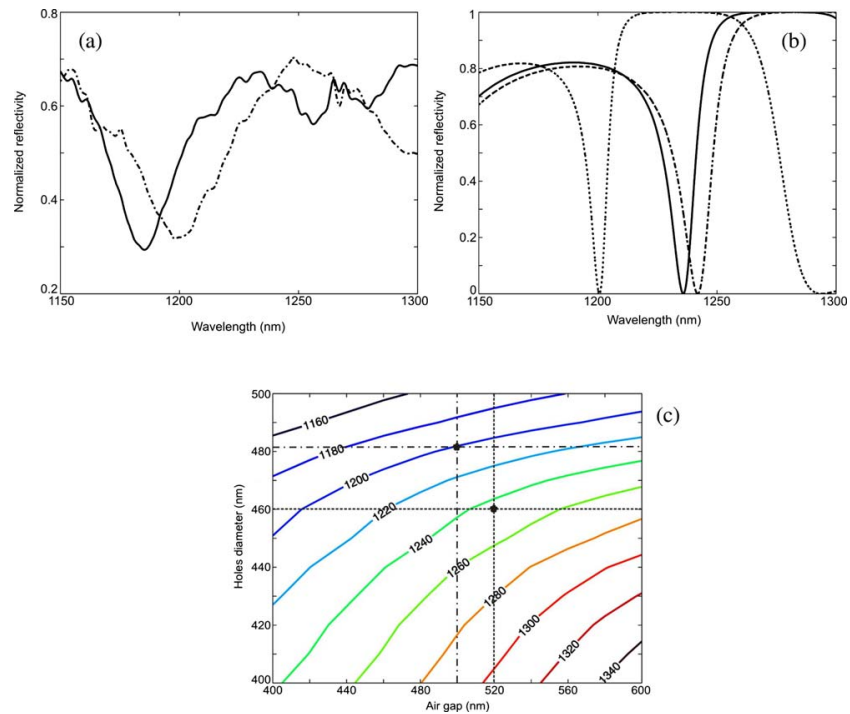

Fig. 3. (Color online) (a) Measured and (b) simulated reflection spectra of the two coupled GaAs-membranes surrounded by air for two different values of hole diameter: $459 \mathrm{~nm}$ (dashed-dotted line) and $464 \mathrm{~nm}$ (continuous line). (c) Contour plot of the resonance wavelength (in nanometers) versus the PhC slabs air gap and the hole diameter calculated by means of 3D FDTD simulations. The dotted lines indicate the nominal geometrical parameters of one of the fabricated devices and the related resonance wavelength obtained through simulations, whereas the dasheddotted lines indicate a hole diameter and an air-gap value (482 and $500 \mathrm{~nm}$, respectively) that can justify the experimental results, by considering a fabrication tolerance of about 5\%; indeed, note that the dotted line in (b), which shows the simulated spectrum in the case of 482 hole diameter and $500 \mathrm{~nm}$ air gap, fits well with the dashed-dotted line in (a). 
observation of the SEM images reveals that the distance between the slabs is not uniform due to the slight bending of the slabs [see Fig. 2(d)]. Moreover, fabrication tolerances, with respect to the nominal geometrical parameters, and the small roughness of the $\mathrm{PhC}$ sidewalls cause scattering and lower reflectivity of the PhC mirrors [16], leading to the broadening of the measured peak and making the cavity reflectivity at resonance larger than zero [17]. Last, but not the least, the measured and simulated wavelength shifts can be addressed to the SEM inspection systematic errors (5\%-10\% of the absolute value) in the hole diameter determination due to different electron detectors or a different contrast between the hole and the edge [18].

Figure 3(c) shows a contour plot of the resonance wavelength, obtained through extensive simulations, as a function of the separation between the slabs and the hole diameter. In particular, FDTD simulations confirmed that the distance between the PhC slabs can be used to tune the position of the resonance. Moreover, this plot can give a clear picture of the effect of technological tolerances on the resonance frequency of the device, allowing one to extrapolate a reasonable set of real geometrical parameters (i.e., hole diameter and air gap) through comparison between simulation and experimental results [16], by considering a realistic fabrication tolerance equal to about $5 \%$.

The dependence of the resonance wavelength on the air gap is linear, and this makes the structure suitable for sensing applications, since a variation of $50 \mathrm{~nm}$ in the distance between the slabs causes a resonance peak shift of roughly $25 \mathrm{~nm}$. A structural strain, stress, and displacement analysis for a single membrane, reported in [19], shows no collapse under such a displacement. This result can be also applied to a double-slab device becoming a straightforward starting point toward a new kind of displacement or pressure sensitive optical device. Moreover, the infiltration of a polymer between the two membranes could improve the uniformity of the separation between the PhC slabs and the mechanical stability of the overall structure.

In conclusion we have demonstrated the fabrication of an ultracompact optical filter based on two coupled PhC GaAs membranes highly sensitive to the material and geometrical parameters. We combined a good resolution of the e-beam writing with a deepetching technique, and this highlights that very high standards of the fabrication accuracy need to be met in order to realize high-quality sensitive $\mathrm{PhC}$ devices. Along this line, the same technology can be applied for fabricating a new class of optical sensing devices based on two coupled $\mathrm{PhC}$ membranes operating in the visible spectral range.

The authors gratefully thank the support of Dr. Antonio Qualtieri and the expert technical help of Gianmichele Epifani. This work was supported by Ministero dell'Istruzione, dell'Università e della RicercaProgetto di Ricerca di Interesse Nazionale 2005, in the framework of the national research project "Photonic Band Gap Nanosensors."

\section{References}

1. T. F. Krauss and R. M. De La Rue, Prog. Quantum Electron. 23, 51 (1999).

2. T. Stomeo, F. Van Laere, M. Ayre, C. Camburnac, H. Benisty, D. Van Thourhout, R. Baets, and T. F. Krauss, Opt. Lett. 33, 884 (2008).

3. M. Grande, L. O'Faolain, T. P. White, M. Spurny, A. D'Orazio, and T. F. Krauss, Opt. Lett. 34, 3292 (2009).

4. K. T. Posani, V. Tripathi, S. Annamalai, N. R. WeisseBernstein, S. Krishna, R. Perahia, O. Crisafulli, and O. J. Painter, Appl. Phys. Lett. 88, 151104 (2006).

5. T. Sünner, T. Stichel, S.-H. Kwon, T. W. Schlereth, S. Höfling, M. Kamp, and A. Forchel, Appl. Phys. Lett. 92, 261112 (2008).

6. E. Chow, A. Grot, L. W. Mirkarimi, M. Sigalas, and G. Girolami, Opt. Lett. 29, 1093 (2004).

7. J. Topol'ancik, P. Bhattacharya, J. Sabarinathan, and P. C. Yu, Appl. Phys. Lett. 82, 1143 (2003).

8. S. G. Johnson, S. Fan, P. R. Villeneuve, J. D. Joannopoulos, and L. A. Kolodzjeski, Phys. Rev. B 62, $8212(2000)$.

9. W. Suh, M. F. Yanik, O. Solgaard, and S. Fan, Appl. Phys. Lett. 82, 1999 (2003).

10. M. Kanskar, P. Paddon, V. Pacradouni, R. Morin, A. Busch, J. F. Young, S. R. Johnson, J. Mackenzie, and T. Tiedje, Appl. Phys. Lett. 70, 1438 (1997).

11. S. Fan and J. D. Joannopoulos, Phys. Rev. B 65, 235112 (2002).

12. M. Notomi, H. Taniyama, S. Mitsugi, and E. Kuramochi, Phys. Rev. Lett. 97, 023903 (2006).

13. L. Shi, P. Pottier, M. Skorobogatiy, and Y. A. Peter, Opt. Express 17, 10623 (2009).

14. L. O'Faolain, M. V. Kotlyar, N. Tripathi, R. Wilson, and T. F. Krauss, J. Vac. Sci. Technol. B 24, 336 (2006).

15. T. Stomeo, V. Errico, A. Salhi, A. Passaseo, R. Cingolani, A. D'Orazio, V. Marrocco, and M. De Vittorio, Microelectron. Eng. 83, 1823 (2006).

16. H. Shin, Y. Ju, H. Song, D. Song, Il-Y. Han, J. Ser, H. Ryu, and Y. Lee, Appl. Phys. Lett. 72, 2205 (1998).

17. B. E. A. Saleh and M. C. Teich, Fundamentals of Photonics (Wiley, 1991).

18. D. M. Beggs, L. O'Faolain, and T. F. Krauss, Photonics Nanostruct. Fundam. Appl. 6, 213 (2008).

19. D. Biallo, A. D'Orazio, M. De Sario, V. Marrocco, V. Petruzzelli, M. A. Vincenti, F. Prudenzano, T. Stomeo, M. Grande, G. Visimberga, R. Cingolani, and M. De Vittorio, J. Eur. Opt. Soc. Rapid Publ. 2, 1 (2007). 